\title{
Ação do Pet - Saúde na Construção de Projetos Terapêuticos Singulares: Relato de Experiência
}

\author{
The actions of "PET-Saúde" in the Development of Singular \\ Therapeutic Projects: An Experience Report
}

\author{
CLARISSIANE SERAFIM CARDOSO ${ }^{1}$ \\ THAMIRES RIBEIRO CHAVES ${ }^{2}$ \\ MARIA JANILCE OLIVEIRA MAGALHÃES ${ }^{3}$ \\ ISABELA LEMOS VELOSO LOPES ${ }^{4}$ \\ TALITHARODRIGUES RIBEIRO FERNANDES PESSOA ${ }^{5}$
}

\section{RESUMO}

Objetivo: Relatar a experiência de acadêmicos e profissionais da área de saúde na construção de Projetos Terapêuticos Singulares (PTS) para portadores de Doenças Crônicas NãoTransmissíveis (DCNT) junto à Estratégia Saúde da Família do bairro do Grotão, Município de João Pessoa-PB, a partir da ação do Programa de Educação pelo Trabalho para Saúde (Pet-Saúde), desenvolvido nos anos de 2013 e 2014, vinculado à Universidade Federal da Paraíba (UFPB) em convênio com a Secretaria Municipal de Saúde (SMS), João Pessoa/PB/Brasil. Relato de Experiência: Refletindo sobre os desafios e contribuições do PET-Saúde na formação profissional e nos serviços de saúde e sabendo do crescente aumento de portadores de Doenças Crônicas NãoTransmissíveis (DCNT) no Brasil, foram construídos Projetos Terapêuticos Singulares favorecendo um trabalho interdisciplinar com a equipe e viabilizando o uso do PTS na Atenção Básica. Os alunos e a equipe de saúde envolvidos no projeto realizaram conversas, ações lúdicas, dieta, entre outras atividades. Sempre de forma interdisciplinar, o PTS junto aos usuários, proporcionou melhorias da qualidade de vida e a mudança por hábitos saudáveis. Conclusões: Observou-se que o PET-Saúde contribuiu com estratégias interdisciplinares na produção do cuidado ao usuário, colaborando de forma pertinente com a formação profissional e criando novas perspectivas ao processo de trabalho dos profissionais de saúde, utilizando para tanto, o PTS como instrumento de intervenção na vida dos portadores de DCNT.

\section{DESCRITORES}

Assistência à Saúde. Atenção à Saúde. Doenças Crônicas.

\begin{abstract}
Objective: To report the experience of university students and health professionals in the development of Singular Therapeutic Projects (STPs) for people with chronic noncommunicable diseases (CNCD). STPs were developed as part of the actions of the "PET-Saúde" (Education Program for Health Work) together with the Family Health Team of Grotão in the city of João Pessoa, PB, between 2013 and 2014. The "PET-Saúde" is a partnership of the Federal University of Paraíba (UFPB) with the Municipal Health Department. Experience Report: STPs were developed taking into account the challenges and contributions of "PET-Saúde" in vocational training and health service and also the increasing number of patients with chronic non-communicable diseases (CNCD) in Brazil. This process favored an interdisciplinary work with the health care team and enabled the use of STPs in primary care. The students and the health professionals embracing this project performed dialogue sections, ludic activities, nutritional orientation, among other activities. Under an interdisciplinary perspective, the STPs improved the users' quality of life and favored a healthy life style. Conclusions: The "PET-Saúde" contributed with interdisciplinary strategies to provide care to the user. In addition, this project impacted positively professional training and the creation of new perspectives on the working process, and STPs were used as an intervention instrument in the lives of people with NCDs.
\end{abstract}

\section{DESCRIPTORS}

Health Care. Chronic Disease. Delivery of Health Care.

Odontóloga, Pesquisadora do Grupo Saúde Coletiva da Universidade Federal de Alagoas/UFAL, Maceió- AL, Brasil.

2 Nutricionista, Mestranda em Ciências da Nutrição com ênfase em Clínica e Epidemiologia da Nutrição pela Universidade Federal da Paraíba (UFPB), Pesquisadora do Núcleo Interdisciplinar de Estudos em Saúde e Nutrição (NIESN / UFPB). João Pessoa-PB, Brasil.

3 Médica da Estratégia Saúde da Família (ESF) do bairro do Grotão, Município de João Pessoa-PB, Brasil.

4 Enfermeira da Estratégia Saúde da Família (ESF) do bairro do Grotão, Município de João Pessoa-PB, Brasil

5 Doutora em Saúde Coletiva pela Universidade Federal do Rio Grande do Norte (UFRN), Docente da Universidade Federal da Paraíba (UFPB). João Pessoa-PB, Brasil. 
$\mathrm{O}$ Sistema Único de Saúde (SUS) objetiva acesso iniversal em todos os níveis de cuidado. A atenção básica vem sendo possibilitada cada vez mais por equipes da Estratégia Saúde da Família (ESF), cuja expansão progressiva melhorou o acesso ao cuidado integral e contínuo à saúde ${ }^{1}$.

A ESF representa um dos principais eixos de ação do Ministério da Saúde para mudar o modelo de assistência à saúde no país. A estratégia prioriza as ações de promoção, proteção e recuperação da saúde dos indivíduos e da família, do recém-nascido ao idoso, sadios ou doentes, de forma integral e/ou contínua 2 .

Nesse contexto, O Programa de Educação pelo Trabalho para a Saúde (PET-Saúde), fruto da ação intersetorial do Ministério da Saúde e Educação, atua através do fomento à formação de grupos de aprendizagem tutorial em áreas estratégicas para o SUS, caracterizando-se como instrumento para qualificação em serviço dos profissionais de saúde, bem como da iniciação ao trabalho e vivências dirigidas aos estudantes da graduação em saúde ${ }^{3}$.

O Pró-PET-Saúde em desenvolvimento nos anos de 2013/2014 na Universidade Federal da Paraíba (UFPB) foi implantado com o objetivo de estimular mudanças curriculares com inserção dos estudantes nas Unidades de Saúde da Família (USF), além de desenvolver processos de formação e educação permanente com os diversos profissionais do serviço; estimular e fortalecer iniciativas de mudança no processo de trabalho em saúde no caminho da integralidade e desenvolver pesquisas com base nas necessidades locais ${ }^{4}$.

Um exemplo é a discussão sobre o crescente aumento das Doenças Crônicas Não-Transmissíveis (DCNT), que tem tido o suporte da Atenção Básica como espaço ideal para o cuidado, o planejamento e a prevenção destas, pois são vistas atualmente como um grande problema de saúde pública que ameaça o desenvolvimento humano ${ }^{5}$.

$\mathrm{Na}$ busca por arranjos e dispositivos que possam ser eficazes para as DCNT, destacam-se os Projetos Terapêuticos Singulares, como sendo "um conjunto de propostas de condutas terapêticas articuladas, para um sujeito individual ou coletivo, resultado da discussão coletiva de uma equipe interdisciplinar" $"$
A proposta do PTS foi desenvolvida com a intenção de proporcionar uma atuação integrada da equipe, em que os diferentes saberes profissionais possam auxiliar na definição de propostas de ações para um sujeito individual dentro da coletividade do serviço. Isto permite que o planejamento do tratamento supere os aspectos do diagnóstico psiquiátrico e da medicação, e inclua a dimensão política, a garantia de direitos, o resgate da cidadania e a reinserção social, como também a dimensão subjetiva e clínica na condução das práticas dirigidas ao usuário ${ }^{7}$.

Este trabalho tem por objetivo relatar experiências vivenciadas por acadêmicos e profissionais de saúde, durante a construção de Projetos Terapêuticos Singulares para usuários portadores de DCNT em uma Unidade de Saúde da Família do bairro do Grotão do Município de João Pessoa-PB.

\section{RELATO DE EXPERIÊNCIA}

O Programa de Educação pelo Trabalho para Saúde (Pet-Saúde) implantado na Universidade Federal da Paraíba (UFPB) e vivenciado na Unidade de Saúde da Família (USF) do Grotão II, no município de João Pessoa-PB, envolveu uma Tutora docente do curso de Odontologia da UFPB, duas Preceptoras, médica e enfermeira da equipe de saúde da família, duas estudantes bolsistas dos cursos de graduação em Nutrição e Odontologia e seis Agentes Comunitários de Saúde (ACS).

Foi visto, portanto, uma boa interdisciplinaridade entre os membros do grupo, e a partir da demanda relatada pelos profissionais de saúde quanto às maiores necessidades da comunidade, surgiu a ideia de atuar de forma pertinente na vida de portadores de Doenças Crônicas Não-Transmissíveis, já que esses usuários se limitavam à procura por assistência à saúde.

Diante da necessidade da busca por estratégias para a adesão dos usuários ao tratamento das DCNT, viabilizou-se a construção de Projetos Terapêuticos Singulares (PTS) no âmbito da ESF.

A experiência vivenciada na USF Grotão II ocorreu durante os anos de 2013 e 2014 e foi inicialmente realizado um mapeamento dos usuários portadores de DCNT na área de abrangência da equipe envolvida. 
Participaram da vivência seis usuários portadores de DCNT, restritos a domicílio e com cuidador profissional e/ou familiar, possibilitando assim uma efetiva intervenção e continuidade no processo de cuidado.

Para a construção do PTS foram desenvolvidos uma ficha clínica de avaliação e um formulário a ser preenchido com o percurso desses usuários na Rede de Atenção à Saúde e com a descrição das atividades de cuidado prestados por parte da equipe de saúde.

Concomitante o desenvolvimento do PTS, foram desenvolvidas ações de Educação Permanente junto à Equipe de Saúde da Família e oficinas para os cuidadores dos usuários.

A partir do contato com a realidade do serviço de saúde, um trabalho coletivo envolvendo tutor, preceptores, estudantes e ACS foi desenvolvido na perspectiva de criar estratégias para a prevenção e cuidados à saúde de usuários portadores de DCNT com maiores fragilidades e demandando uma atenção integral da equipe. Nesse sentido, a construção dos PTS foi estudada, estruturada e elaborada.

O grupo tutorial Saúde da Família e Redes do PET-Saúde UFPB elaborou um questionário estruturado no qual continham informações sobre condições socioeconômicas, arranjo familiar, histórico de doenças, condições de saúde e expectativas em relação à situação vivida. A anamnese realizada possibilitou uma avaliação das vulnerabilidades do usuário e o contexto ao qual estava inserido, de forma que a partir dessas informações, pôde-se estipular metas a serem alcançadas, quem as realizariam, as articulações possíveis com as Redes de atenção e o período de realização.

Para a aplicação do questionário foram realizadas visitas domiciliares utilizando uma abordagem integral ao usuário, que com a atuação das estudantes de nutrição e odontologia, os cuidados com a alimentação e a prevenção e tratamento da saúde bucal foram abordagens constantes. No início, foram necessárias conversas para explicar e sensibilizar os usuários e famílias quanto à prática do PTS.

A sensibilização para o trabalho também foi necessária à equipe de saúde. Para tanto, as estudantes em articulação com as preceptoras da USF realizaram uma palestra para os membros da equipe, sobretudo para os ACS, antes do início da construção dos PTS. A conversa se fez necessária para a continuidade do processo de mudanças quanto ao cuidado dos usuários na forma proposta no projeto terapêutico. Percebeu-se uma grande resistência inicial que se caracterizava como mais uma demanda a ser realizada. Porém, após um início sem colaborações por parte da equipe, a exposição da proposta como benéfica a todos os atores envolvidos contribuiu para uma maior aceitação por parte dos profissionais.

Após o início da construção dos PTS, e envolvimento da família, usuário e equipe, buscou-se garantir continuidade ao processo. Os membros do PETSaúde motivaram a equipe de saúde quanto à adesão dos PTS, sendo corresponsáveis junto à família. Foram destacadas as mudanças no perfil situacional de cada usuário como motivo para continuação e estimulo à realização de outros PTS.

As ações do PTS foram realizadas por meio de abordagens da rotina clínica (verificação de pressão, glicemia capilar, aferição de peso, checagem de sinais vitais, ausculta cardíaca, prescrição de medicação, encaminhamento de exames, outros), além de avaliação nutricional com aferição de peso e medidas, orientação nutricional e proposta de cardápio, bem como avaliação odontológica tanto de caráter preventivo como de tratamento.

Um aspecto relevante foi o desenvolvimento de estratégias para a formação de vínculo e colaboração por parte dos usuários. Desta forma, atividades lúdicas que envolveram música também trouxeram uma resposta benéfica ao qual o usuário relatava bem-estar e estimulo a continuar com o projeto. Uma das estudantes levava um violão, e as preceptoras instrumentos de percussão, enquanto os outros utilizavam a voz e gestos para animar a visita que realizavam à casa do usuário. Ao final de cada visita, quando a equipe buscava não apenas realizar os cuidados de saúde, mas estimular que a família também participasse, acontecia o momento musical, onde todos os sujeitos envolvidos participavam ativamente.

Nesta experiência foram construídos seis PTS, e pôde-se observar o envolvimento do usuário com o processo de saúde ao qual estava inserido, e a colaboração da equipe, ainda que de forma tímida. As estratégias e intervenções dos profissionais da equipe geraram trocas de saberes e propiciaram uma mudança na perspectiva da atenção voltada ao usuário. 


\section{CONCLUSÕES}

Com o desenvolvimento dos Projetos Terapêuticos Singulares em uma Unidade de Saúde da Família pôde-se observar a contribuição para a coletividade da produção do cuidado, com a criação de vínculo e uma responsabilização com o usuário garantindo uma atenção continuada. Existindo assim, a necessidade de disparar mudanças nas práticas de saúde diversificando as ofertas de serviços de saúde, provocar processos de reflexão nos profissionais possibilitando repensarem seu processo de trabalho, suas práticas e a instituição na qual estão inseridos.

Na perspectiva das DCNT, o PTS surgiu como mais uma estratégia de combate e prevenção, onde o usuário ao ser sujeito ativo foi também capacitado a lidar com seu processo de saúde.

A colaboração do PET-Saúde em viabilizar um espaço para discussão e construção do PTS trouxe a vivência interdisciplinar e planejada, aliada à atuação

\section{REFERÊNCIAS}

1. Schmidt MI, Menezes PR, Chor D, Barreto SM, Monteiro $A A$, Menezes AM, et al. Doenças crônicas não transmissíveis no Brasil: carga e desafios atuais. The Lancet [Internet]. 2011; 377 (9781) : 1949-196

2. Schmidt MI, Duncan BB, Stevens A, Luft V, Iser BPM, Moura $\mathrm{L}$, et al. Doenças crônicas não transmissíveis no Brasil: mortalidade, morbidade e fatores de risco. In: Saúde Brasil 2009: uma análise da situação de saúde e da Agenda Nacional e Internacional de Prioridades em Saúde. Ministério da Saúde, Secretaria de Vigilância em Saúde, Departamento de Análise de Situação de Saúde. - Brasília : Ministério da Saúde, 2010: 111-36.

3. Ministério da Saúde. Portaria Interministerial n ${ }^{\circ} 421$, de 3 de março de 2010. Institui o Programa de Educação pelo Trabalho para a Saúde (PET Saúde) e dá outras providências. Diário Oficial da União, Brasília, DF; 2008.

4. Galvão MHR, Freitas CHSM, Cassemiro LL, PereiraPsicológa IL, Oliveira MG. PET-saúde: gestão e atenção à saúde potencializando mudanças na formação. Revista da ABENO. 2014; 14(1):57-65. em diferentes contextos sociais, contribuindo para a formação profissional das estudantes, bem como a colaboração para a realização das ações e metas traçadas no PTS. Além de trazer aos profissionais novas perspectivas de atuação dentro do serviço de saúde, e levá-los a um pensamento crítico-reflexivo do processo de trabalho ao qual estão inseridos.

Apesar das dificuldades de articulação com a equipe em adaptar as demandas do serviço à construção do PTS, viabilizar este dispositivo é recomendado às equipes de saúde, como forma de garantir a continuidade da avaliação e da reavaliação do processo terapêutico, em menor espaço de tempo possível. Enfatiza-se a construção do PTS como atividade rotineira a ser desenvolvida nos serviços de saúde na atenção básica, uma vez que o mesmo busca atender a demandas de saúde complexas e com isso, uma abordagem interdisciplinar articulando saberes e trocas de conhecimento, possibilitando a autonomia ao usuário, tornando-o sujeito ativo na construção da sua saúde.

5. Capilheira M, Santos IS. Doenças crônicas não transmissíveis: desempenho no cuidado médico em atenção primária à saúde no sul do Brasil. Cad. Saúde Pública. 2011; 27(6):1143-1153.

6. Ministério da Saúde. Humaniza SUS: documento-base para gestores e trabalhadores do SUS. $4^{a}$ ed. Brasília, DF: Editora do Ministério da Saúde; 2008.

7. Ministério da Saúde. Cartilha do Ministério da Saúde: Clínica Ampliada, Técnico de Referência e Projeto Terapêutico Singular. $2^{a}$ ed. Brasília, DF: Editora do Ministério da Saúde; 2007.

Correspondência

Clarissiane Serafim Cardoso

Universidade Federal de Alagoas

Avenida Lourival Melo Mota, s/n

Cidade Universitária, Campus A. C. Simões

CEP: 57072-900 - Maceió - Alagoas - Brasil

E-mail: clarinha_16_@hotmail.com 